pute the possibility that CD38-deficient neutrophils could be defective in other functional assays. Thus, if $\beta_{2}$ integrininduced $\mathrm{Ca}^{++}$release is shown to be cADPR dependent, it would represent a good candidate for another CD38-dependent signaling process in neutrophils.

1. Partida-Sánchez, S. et al. Cyclic ADP-ribose production by $\mathrm{CD} 38$ regulates intracellular calcium release, extracellular calcium influx and chemotaxis in neutrophils and is required for bacterial clearance in vivo. Nature Med. 7, 1209-1216 (2001).

2. Murphy, P.M. The molecular biology of chemoattractant receptors. Ann. Rev. Immunol. 12, 593-633 (1994).

3. Scharff, O. \& Foder, B. Regulation of cytosolic calcium in blood cells. Physiol. Rev. 73, 547-582 (1993).

4. Davies-Cox, E.V., Laffafian, I. \& Hallett, M.B. Control of $\mathrm{Ca}^{2+}$ influx in human neutrophils by inositol 1,4,5-trisphosphate $\left(\mathrm{IP}_{3}\right)$ binding: Differential effects of micro-injected $\mathrm{IP}_{3}$ receptor receptor antagonists. Biochem. J. 355, 139-143
(2001).

5. Li, Z., et al. Roles of PLC- $\beta 2$ and $-\beta 3$ and PI3K $\gamma$ in chemoattractant mediated signal transduction. Science 287, 1046-1049 (2000).

6. Cancela, J.M. Specific $\mathrm{Ca}^{2+}$ signaling evoked by cholesystokinin and actetylcholine: the roles of NAADP, CADPR and IP3. Ann. Rev. Physiol. 63 99-117 (2001).

7. Laffafian, I. \& Hallett, M.B. Does cytosolic free $\mathrm{Ca}^{2+}$ signal neutrophil chemotaxis? J. Cell Sci. 108 3199-3205 (1995).

8. Fallman, M., Andersson, R. \& Andersson, T. Signaling properties of CR3 (CD11b/CD18) and CR1(CD35) in relation to phagocytosis of complement-opsonised particles. J. Immunol. 151, 330-338 (1993).

9. Petersen, M., Williams, J.D. \& Hallett, M.B. Crosslinking of $C D 11 \mathrm{~b}$ or CD18 signals release of localised $\mathrm{Ca}^{2+}$ from intracellular stores in neutrophils. Immunology 80, 157-159 (1993).

10. Laffafian, I. \& Hallett, M.B. Lipid-assisted microinjection: Introducing material into the cytosol and membranes of small cells. Biophys J. 75, 2558-2563 (1998).

11. Gao, J.-L., Lee, E.J. \& Murphy, P.M. Impaired antibacterial host defense in mice lacking the $\mathrm{N}$ formylpeptide receptor. J. Exp. Med. 189, 657-662 (1999)
12. Alemany, R., Meyer zu Herigndorf, D., van Koppen, C.J. \& Jakobs, K.H. Formyl peptide receptor signaling in HL-60 cells through sphingosine kinase. I. Biol. Chem. 274, 3994-3999 (1999).

13. Lee, H.C. Potentiation of calcium- and caffeineinduced calcium release by cyclic ADP-ribose. I. Biol. Chem. 268, 293-299 (1993).

14. Galione, A. \& White, A. $\mathrm{Ca}^{2+}$ release induced by cyclic ADP-ribose. Trends Cell Biol. 4, 431-436 (1994).

15. Kiselyov, K.I. et al. Gating of store-operated channels by conformational coupling to ryanodine receptors. Mol. Cell 6, 421-431 (2000).

16. Hirsch, E. et al. Central role for $G$ protein-coupled phosphoinositide 3-kinase $\gamma$ in inflammation. Science 287, 1049-1053 (2000).

17. Sasaki, T. et al. Function of $\mathrm{P} 13 \mathrm{~K} \gamma$ in thymocyte development, $\mathrm{T}$ cell activation, and neutrophil migration. Science 287, 1040-1046 (2000).

Frances E. Lund, Troy D. Randall \& SANTIAGO PARTIDA-SÁNCHEZ

Trudeau Institute

Saranac Lake, New York, USA

Email: flund@trudeauinstitute.org

\title{
Dyslipidemia due to retroviral protease inhibitors
}

To the editor-The biochemical basis for the development of lipodystrophy in HIV-1-infected patients treated with retroviral protease inhibitors (PIs) is unclear. Liang et al. ${ }^{1}$ demonstrate that the PIs ritonavir and saquinavir affect fat metabolism through alterations in neutral lipid synthesis and the secretion of apolipoprotein B (ApoB). The authors also propose that inhibition of proteasomal chymotryptic activity by ritonavir and saquinavir contributes to the observed intracellular accumulation of ApoB in vitro. Whereas the latter effect requires drug concentrations in the region of $50-100 \mu \mathrm{M}$, the former effects occur at 5-15 $\mu \mathrm{M}$. For several reasons, we question the relevance of effects on proteasome function to the development of metabolic abnormalities associated with PI-induced lipodystrophy in vivo.

First, although we agree that ritonavir and saquinavir inhibit the chymotryptic activity of isolated $20 \mathrm{~S}$ proteasomes in vitro, two other HIV-1 PIs, indinavir and nelfinavir, do not ${ }^{2,3}$. The use of either indinavir or nelfinavir would therefore have provided relevant controls for the experiments attributing intracellular accumulation of ApoB to inhibition of proteasomal activity. Further, although ritonavir does seem particularly likely to induce severe dyslipidemia, this metabolic complication of therapy is a feature of all retroviral PIs. Thus, it is difficult to attribute PI-associated lipodystrophy primarily to proteasome inhibition.

Second, the authors claim that the effects on ApoB metabolism attributed to inhibition of the proteasome were seen at therapeutically relevant concentrations of ritonavir and saquinavir. The in vitro $\mathrm{IC}_{90} \quad(90 \%$ inhibitory concentration) of ritonavir is approximately $100 \mathrm{nM}$ and that of saquinavir is less than $50 \mathrm{nM}$ (ref. 4). Maximum plasma concentrations $\left(\mathrm{C}_{\max }\right)$ of these drugs in vivo are also well below the levels used by Liang et al. The $\mathrm{C}_{\max }$ of ritonavir is approximately $15 \mu \mathrm{M}$ (ref. 4). The $\mathrm{C}_{\max }$ for saquinavir, even in ritonavir-boosted regimens, is less than $10 \mu \mathrm{M}$, and in non-boosted regimens is 10 -fold lower ${ }^{5}$. Although hepatocytes might be exposed to higher drug concentrations before systemic redistribution, such exposure is likely to be transient. Moreover, as both drugs are highly protein bound, any extrapolation of drug effects from in vitro to in vivo must take into account differences in protein concentrations. For any given total drug concentration, the pharmacologically active free drug levels are likely to be significantly higher in vitro, where serum concentrations are typically around $10 \%$.

Third, we have found that concentrations greater than $10 \mu \mathrm{M}$ of ritonavir and saquinavir compromise the viability of several B- and T-cell lines in $v$ vitro $^{3}$. The validity of drug-mediated cellular effects detected under such conditions is questionable.

The inhibition of both neutral lipid synthesis and ApoB secretion reported by Liang et al. occurs at concentrations of ritonavir and saquinavir that are feasible in vivo. Interestingly, the authors state that indinavir also causes similar alterations to neutral lipid synthesis. It may be that these in vitro drug effects provide insight into the generation of the lipid abnormalities that complicate the treatment of HIV-1 infected individuals with retroviral PIs. We would, however, caution against overemphasizing the role of proteasome inhibition in the development of lipodystrophy for the reasons outlined above.

Anthony D. Kelleher ${ }^{1}$, Andrew K. Sewell ${ }^{2} \&$ David A. Price ${ }^{3}$

${ }^{1}$ National Centre in HIV Epidemiology and

Clinical Research, Darlinghurst, NSW,

Australia

${ }^{2}$ The Peter Medawar Building for Pathogen Research, South Parks Road, Oxford, UK ${ }^{3}$ Nuffield Department of Clinical Medicine, John Radcliffe Hospital, Oxford, UK

Sturley et al. reply-The major issue raised by Kelleher et al. regards the pharmokinetics of HIV protease inhibitors such as 